\title{
Response to Neeleman et al.
}

To the Editor:

We read with great interest the research letter to the editor by Neeleman and colleagues on the use of heme arginate as the initial treatment for severe neuromuscular decompensation in tyrosinemia type I (HT-1). ${ }^{1}$ The authors would like to suggest that it be added to recent treatment guidelines for HT-1, including one by ourselves, as a possible life-saving treatment. $^{2}$ Three most recent treatment (HT-1) guidelines referenced in their text did not include this suggestion. ${ }^{2-4}$ There have been few anecdotal reports of this therapy since current treatment regimens all emphasize ensuring the reinitiation of nitisinone and symptomatic treatment of the skeletal muscle pain, accompanying hypertension and hyponatremia if present. Intravenous (IV) treatment with glucose should decrease the porphyrin pathway production of the neurologic offending 5-amino-levulinic acid (5-ALA) and reinitiation of nitisinone, the required chronic treatment for HT-1, should be immediately begun to prevent the continued accumulation of succinylacetone blocking the heme synthetic pathway that results in 5-ALA accumulation. Giving heme initially alone may temporarily address the chemical causation of the symptoms (5-ALA) but will not address the actual pathophysiologic problem of HT-1, i.e., the effect of ongoing succinylacetone accumulation on liver and renal functions and its direct effect on the heme biosynthetic pathway.

Heme therapy in HT-1 has not been evaluated rigorously. The major reason to currently minimize the use of heme is that there is insufficient published data on its use and effectiveness compared with nitisinone and IV glucose/saline support. Heme may be difficult to obtain quickly and not every medical setting will have availability of 5-ALA laboratory results in a timely manner. Based on our discussion within the physician group who coauthored the guidelines described in Chinsky et al., ${ }^{2}$ it was determined from group experience that the multiple episodes that we treated associated with neuromuscular symptoms, hyponatremia, and hypertension were all successfully handled by using IV glucose containing normal saline, antihypertensive drugs, antiemetics such as ondansetron, and pain control medications as needed. ${ }^{2}$ Most importantly, nitisinone should be restarted as soon as possible. We did not want to comment on theoretical or anecdotal treatments without clear published results. Neeleman et al. ${ }^{1}$ now provide one such recent case where nitisinone was available but there are issues using this case as the example to recommend hemebased treatment. ${ }^{1}$ There was no hyponatremia (sodium 136) with the third episode where heme was used, and blood pressures are not described for interpretation of this being a severe attack. Neuromuscular signs and symptoms (paresthesias in case 3) and compromised oral route (nausea and vomiting) were the reasons given for direct use of IV heme rather than oral nitisinone but there is no mention of antiemetic (i.e., ondansetron or similar) use. However, Fig. 1 in Neeleman et al. ${ }^{1}$ suggests that nitisinone was used at some point concurrently with the heme arginate. Similarly, no mention of the use of IV glucose containing normal saline is made and certainly this could have been the reason for the initial decline in porphyrin pathway activity that aided recovery if it was indeed used.

There are now at least two commercial preparations of nitisinone that allow liquid form to be ingested (one aqueous suspension, one glycerin based), and it is likely that the vast majority, if not all, of these types of patients can be treated and recover without resorting to starting heme infusion therapy. One recent published case of a severe neuromuscular episode with vomiting being treated without resorting to heme therapy certainly provides another strong anecdotal cases (Rank et al.) ${ }^{6}$ that contradicts the conclusion of Neeleman et al. to use heme infusion as initial therapy. ${ }^{5}$ Heme infusion therapy is not without its risks, such as cessation of therapy due to a coagulopathy requiring exchange transfusion that developed being described for one of the anecdotal cases (Rank et al.) ${ }^{6}$ described by Neeleman et al. ${ }^{1}$ Heme also may not be as immediately available as the patient's own nitisinone, and the other approaches described herein are available wherever acute hospital care is sought. Acquisition of nitisinone for emergency use from the known commercial distributor for the patient would likely be as quick as acquisition of heme if the patient's family cannot supply the medication.

For extreme cases that fail to respond after several days of the prior published therapy recommended, perhaps use of heme can be considered but should be thought of similar to a research-based, institutional review board-approved approach. At the present, first-line therapy for neuromuscular episodes should not be associated with heme approaches except perhaps under very extreme circumstances, certainly not the patient examples given in Neeleman et al. ${ }^{1}$ The authors do raise a valid point to consider, but only after more conventional advocated therapies have been initiated and/or cannot be used.

\section{DISCLOSURE}

J.M.C., C.F., and C.R.S. have been intermittent consultants and lecturers for Sobi, Inc. J.M.C. and C.F. have been intermittent consultants and lecturers for Cycle Pharmaceuticals, Inc. Both companies are manufacturers of forms of nitisinone described in this paper but neither group had any input or knowledge of this paper prior to submission for publication. 
Publisher's note Springer Nature remains neutral with regard to jurisdictional claims in published maps and institutional affiliations.

Jeffrey M. Chinsky, MDPhD (1), Can Ficicioglu, $\mathrm{MD} P \mathrm{Ph}^{2}$ and C. Ronald Scott, $M D^{3}$

${ }^{1}$ Department of Pediatrics, Johns Hopkins University School of Medicine, Baltimore, MD, USA; ${ }^{2}$ The Children's Hospital of Philadelphia, Division of Human Genetics and Metabolism, Perelman School of Medicine at The University of Pennsylvania, Philadelphia, PA, USA; ${ }^{3}$ Division of Genetic Medicine, Department of Pediatrics, University of Washington School of Medicine, Seattle, WA, USA. Correspondence: Jeffrey M. Chinsky (Jchinsky1@gmail.com)

\section{REFERENCES}

1. Neeleman RA, Wilson JHP, Williams M, Langendonk JG Heme as an initial treatment for severe decompensation in tyrosinemia type I. Genet Med (in press).
2. Chinsky JM, Singh R, Ficicioglu C, et al. Diagnosis and treatment of tyrosinemia type I: a US and Canadian consensus group review and recommendation. Genet Med. 2017;19:1380.

3. de Laet C, Dionisi-Vici C, Leonard JV, et al. Recommendations for the management of tyrosinaemia type 1. Orphanet J Rare Dis. 2013;8:8.

4. Geppert J, Stinton C, Freeman K, et al. Evaluation of presymptomatic nitisinone treatment on long-term outsomes in tyrosinemia type 1 patients: a systematic review. Orphanet J Rare Dis. 2017;12:154.

5. Yazici H, Canda E, Esra ER, et al. Tyrosinemia type 1 and reversible neurogenic crisis after one month interruption of nitisinone. J Pediatr Res. 2018;5 suppl 1:57-59.

6. Rank JM, Pascual-Leone A, Payne W, et al. Hematin therapy for the neurologic crisis of tyrosinemia. J Pediatr. 1990;118:136-139.

Advance online publication 1 October 2019. doi:10.1038/s41436-019-0659-y 